\title{
The Asymptotic Inversion of Certain Cumulative Distribution Functions
}

\author{
A. Gil ${ }^{1}$, J. Segura ${ }^{2}$ and N.M. Temme ${ }^{3}$
}

1 Departamento de Matemática Aplicada y Ciencias de la Computación, ETSI Caminos, Canales y Puertos, Universidad de Cantabria, 39005-Santander, Spain

2 Departamento de Matemáticas, Estadística y Computación, Universidad de Cantabria, 39005 Santander, Spain

3 CWI, PO Box 94079, 1090 GB Amsterdam, The Netherlands

Summary. The inversion of cumulative distribution functions is an important topic in statistics, probability theory and econometrics, in particular for computing percentage points of the distribution functions. The numerical inversion of these distributions needs accurate starting values, and for the standard distributions powerful asymptotic formulas can be used to obtain these values.. It is explained how a uniform asymptotic expansions of a standard form representing several well-known distribution functions can be used for the asymptotic inversion of these functions. As an example we consider the inversion of the hyperbolic cumulative distribution function.

\section{Introduction}

We consider functions of the form

$$
F_{a}(\eta)=\sqrt{\frac{a}{2 \pi}} \int_{-\infty}^{\eta} e^{-\frac{1}{2} a \zeta^{2}} f(\zeta) d \zeta
$$

where $a>0, \eta \in \mathbb{R}$, and $f$ is analytic and real on $\mathbb{R}$ with $f(0)=1$.

The special case $f=1$ gives the normal distribution

$$
P(\eta \sqrt{a})=\sqrt{\frac{a}{2 \pi}} \int_{-\infty}^{\eta} e^{-\frac{1}{2} a \zeta^{2}} d \zeta=\frac{1}{2} \operatorname{erfc}(-\eta \sqrt{a / 2}),
$$

where $\operatorname{erfc} z$ is the complementary error function

$$
\operatorname{erfc} z=\frac{2}{\sqrt{\pi}} \int_{z}^{\infty} e^{-t^{2}} d t
$$

As shown in [5], [6] and [3, Chapter 10] the incomplete gamma functions and the incomplete beta function - which are the basic functions for several 
distribution functions - can be written in this form. In these references we have used uniform asymptotic expansions for inverting these distribution functions for large values of one or two parameters.

We explain how the incomplete gamma function

$$
P(a, x)=\frac{1}{\Gamma(a)} \int_{0}^{x} t^{a-1} e^{-t} d t
$$

can be written in the standard form (1). Let $\lambda=\frac{x}{a}$ and $t=a \tau$. Then

$$
\Gamma^{*}(a) P(a, x)=\sqrt{\frac{a}{2 \pi}} \int_{0}^{\lambda} e^{-a(\tau-\ln \tau-1)} \frac{d \tau}{\tau},
$$

where

$$
\Gamma^{*}(a)=\Gamma(a) a^{-a} e^{a} \sqrt{\frac{a}{2 \pi}}=1+\mathcal{O}(1 / a) .
$$

The transformation

$$
\tau-\ln \tau-1=\frac{1}{2} \zeta^{2}, \quad \operatorname{sign}(\tau-1)=\operatorname{sign}(\zeta)
$$

gives the standard form

$$
\Gamma^{*}(a) P(a, x)=\sqrt{\frac{a}{2 \pi}} \int_{-\infty}^{\eta} e^{-\frac{1}{2} a \zeta^{2}} f(\zeta) d \zeta, \quad f(\zeta)=\frac{1}{\tau} \frac{d \tau}{d \zeta}
$$

with

$$
\lambda-\ln \lambda-1=\frac{1}{2} \eta^{2}, \quad \operatorname{sign}(\lambda-1)=\operatorname{sign}(\eta) .
$$

\section{Asymptotic representation of $F_{a}(\eta)$}

By using Laplace's asymptotic method (see [8, Chapter 2]) it is not difficult to find the asymptotic estimates for large positive $a$ and fixed values of $\eta$ :

$$
F_{a}(\eta)= \begin{cases}-f(\eta) /(\eta \sqrt{2 a \pi}) e^{-a \eta^{2}}[1+\mathcal{O}(1 / a)], & \text { if } \eta<0 \\ \frac{1}{2}+\mathcal{O}(1 / \sqrt{a}), & \text { if } \eta=0 \\ 1+\mathcal{O}(1 / a), & \text { if } \eta>0\end{cases}
$$

We see that the asymptotic behaviour of $F_{a}(\eta)$ is completely different in the three cases distinguished. Moreover, the asymptotic forms do not pass into each other when $\eta$ changes sign. By using an integration by parts procedure we can obtain a single asymptotic representation of $F_{a}(\eta)$ which is valid for all $\eta \in \mathbb{R}$. We write in $(1) f(\eta)=[f(\eta)-f(0)]+f(0)$, where $f(0)=1$, and use $(2)$. Then we obtain by repeating integration by parts steps: 


$$
F_{a}(\eta)=\frac{1}{2} \operatorname{erfc}(-\eta \sqrt{a / 2}) F_{a}(\infty)+\frac{e^{-\frac{1}{2} a \eta^{2}}}{\sqrt{2 \pi a}} S_{a}(\eta),
$$

where, as $a \rightarrow \infty$,

$$
F_{a}(\infty) \sim \sum_{n=0}^{\infty} \frac{A_{n}}{a^{n}}, \quad A_{0}=1, \quad S_{a}(\eta) \sim \sum_{n=0}^{\infty} \frac{C_{n}(\eta)}{a^{n}},
$$

uniformly with respect to $\eta \in \mathbb{R}$. The coefficients follow from the following recursive scheme. Let $f_{0}(\eta)=f(\eta)$. Then, for $n=0,1,2, \ldots$, define

$$
f_{n+1}(\eta)=\frac{d}{d \eta} \frac{f_{n}(\eta)-f_{n}(0)}{\eta},
$$

and we have

$$
A_{n}=f_{n}(0), \quad C_{n}(\eta)=\frac{f_{n}(0)-f_{n}(\eta)}{\eta} .
$$

\section{The asymptotic inversion method}

Let $p \in(0,1)$ and $a$ a large positive parameter. Then we are interested in the value $\eta$ that solves the equation

$$
F_{a}(\eta)=F_{a}(\infty) p .
$$

We use the representation in (11) and define a number $\eta_{0}$ that solves the reduced equation

$$
\frac{1}{2} \operatorname{erfc}\left(-\eta_{0} \sqrt{a / 2}\right)=p .
$$

Then for the requested value $\eta$ we assume the expansion

$$
\eta \sim \eta_{0}+\frac{\eta_{1}}{a}+\frac{\eta_{2}}{a^{2}}+\frac{\eta_{3}}{a^{3}}+\ldots, \quad a \rightarrow \infty,
$$

and try to find the coefficients $\eta_{1}, \eta_{2}, \eta_{3}, \ldots$. To obtain the $\eta_{j}$ we can substitute the expansion for $\eta$ into (11) and use formal power series manipulations. For the asymptotic inversion of the incomplete gamma and beta functions we have used techniques based on differential equations; see [5], [6] and [3, Chapter 10]. In the next section we consider a different example.

The method based on differential equations runs as follows. From (1), (15) and (16) we obtain

$$
\frac{d p}{d \eta_{0}}=\sqrt{\frac{a}{2 \pi}} e^{-\frac{1}{2} a \eta_{0}^{2}}, \quad \frac{d p}{d \eta}=\sqrt{\frac{a}{2 \pi}} \frac{f(\eta)}{F_{a}(\infty)} e^{-\frac{1}{2} a \eta^{2}},
$$

from which we obtain, upon dividing,

$$
f(\eta) \frac{d \eta}{d \eta_{0}}=F_{a}(\infty) e^{\frac{1}{2} a\left(\eta^{2}-\eta_{0}^{2}\right)}
$$


Substituting (17) we obtain for $\eta_{1}$ after perturbation analysis in first order for large $a$

$$
f\left(\eta_{0}\right)=e^{\eta_{0} \eta_{1}} \quad \Longrightarrow \quad \eta_{1}=\frac{1}{\eta_{0}} \ln f\left(\eta_{0}\right) .
$$

For higher order terms $\eta_{j}, j \geq 2$, we need in (19) more coefficients in the asymptotic expansion of $F_{a}(\infty)$ (see (12), (30) and (31)) and we have to expand

$$
f(\eta)=f\left(\eta_{0}\right)+\left(\eta-\eta_{0}\right) f^{\prime}\left(\eta_{0}\right)+\frac{1}{2}\left(\eta-\eta_{0}\right)^{2} f^{\prime \prime}\left(\eta_{0}\right)+\ldots
$$

\section{The hyperbolic cumulative distribution}

The hyperbolic distribution was introduced in [2] and is given by

$$
F(y)=C \int_{-\infty}^{y} e^{-\alpha \sqrt{\delta^{2}+(x-\mu)^{2}}+\beta(x-\mu)} d x, \quad y \in \mathbb{R}
$$

where $\alpha>0,|\beta|<\alpha, \delta$ and $\mu$ are arbitrarily real constants, and $C$ is the normalizing constant which gives $F(\infty)=1$. The value of $C$ is given by

$$
C=\frac{\omega}{2 \alpha \delta^{2} K_{1}(\omega)}, \quad \omega=\delta \sqrt{\alpha^{2}-\beta^{2}},
$$

where $K_{1}(\omega)$ denotes the modified Bessel function of the third kind of order 1 (see [1, Chapter 9] or [7, Chapter 9]).

\subsection{A few transformations}

We transform the function $F(y)$ into the standard form. Because $|\beta|<\alpha$, we can write $\beta=\alpha \tanh \theta$. We substitute in (22) $x=\mu+\delta \sinh (\theta+t)$, and obtain

$$
F(y)=\frac{1}{2 K_{1}(\omega)} \int_{-\infty}^{\tau} e^{-\omega \cosh t} \frac{\cosh (t+\theta)}{\cosh \theta} d t
$$

where $\omega$ is given in $(23)$ and

$$
\tau=\operatorname{arcsinh} \frac{y-\mu}{\delta}-\theta, \quad \cosh \theta=\frac{\alpha}{\sqrt{\alpha^{2}-\beta^{2}}} .
$$

Next we use the transformation

$$
\cosh t=1+2 \zeta^{2}, \quad \Longrightarrow \quad t=2 \operatorname{arcsinh} \zeta
$$

which gives

$$
F(y)=\frac{e^{-\omega}}{K_{1}(\omega)} \int_{-\infty}^{\eta} e^{-\frac{1}{2} a \zeta^{2}} f(\zeta) d \zeta
$$


where

$$
a=4 \omega, \quad \eta=\sinh \frac{1}{2} \tau
$$

and

$$
f(\zeta)=\frac{1+2 \zeta^{2}+2 \tanh \theta \zeta \sqrt{\zeta^{2}+1}}{\sqrt{\zeta^{2}+1}} .
$$

We see that $f(0)=1$ and it follows that we can write $F(y)$ in the form

$$
F(y)=\frac{F_{a}(\eta)}{F_{a}(\infty)}, \quad F_{a}(\infty)=\sqrt{\frac{2 \omega}{\pi}} e^{\omega} K_{1}(\omega),
$$

where $F_{a}(\eta)$ has the standard form (1). We have (see [1, Eq. 9.7.2])

$$
F_{a}(\infty)=1+\frac{3}{8 \omega}+\mathcal{O}\left(1 / \omega^{2}\right), \quad \omega \rightarrow \infty .
$$

It follows also that the inversion problem $F(y)=p$ when $a$ is large can be written in the form (15). When we have found $\eta$ from the expansion (17), we compute $\tau=2 \operatorname{arcsinh} \eta$ and finally (see (25))

$$
y=\mu+\delta \sinh (\theta+\tau), \quad \theta=\operatorname{arctanh} \frac{\beta}{\alpha} .
$$

\subsection{A numerical example}

When $a$ is large the function $F_{a}(\eta)$ approaches the unit step function and the numerical inversion needs accurate starting values for, say, Newton's method, in particular when in (15) $p$ is very small or very close to unity.

In [4] analytic approximations for these $p$-values are constructed of the inverse function $F^{-1}$ of $F(y)$ given in (22). With these approximations a numerical algorithm from Mathematica is used to compute the inverse $F^{-1}$ from the differential equation satisfied by this function.

We demonstrate our approach by taking $\alpha=5, \beta=3, \mu=0$ and $\delta=$ $1,10,100$. These values give $\omega=4,40,400$ and $a=16,160,1600$, respectively.

First we compute $\eta_{0}$ from (16) and next $\eta_{1}$ from (20), with $f(\eta)$ given in (29). The computed value $\eta$ then follows from (17) (with two terms). Next we compute $\tau=2 \operatorname{arcsinh} \eta$ (see (28)), and with $\tau$ we can compute $y$ by inverting the second equation in $(25)$ with $\theta=\operatorname{arctanh}(\beta / \alpha)$.

In Table 1 we give for several values of $p$ and $\delta$ the computed value $y$, and the relative error $|F(y)-p| / p$. We observe that the approximations of $y$ become indeed better when the large parameter $a=4 \delta \sqrt{\alpha^{2}-\beta^{2}}$ increases. Also, the approximations are better when $p \sim 1$.

\section{Acknowledgment}

The authors acknowledge financial support of the Spanish Ministerio de Educacion y Ciencia, project MTM200609050. 
Table 1. Values $y$ and relative errors $\Delta=|F(y)-p| / p$ of the inversion $F(y)=p$, where $F(y)$ is given in (22) for $\alpha=5, \beta=3, \mu=0$, and several values of $\delta$ and $p$.

\begin{tabular}{lrccccc} 
& $\delta$ & 1 & & 10 & & \multicolumn{1}{c}{100} \\
\hline \multicolumn{1}{c}{$p$} & $y$ & $\Delta$ & $y$ & $\Delta$ & $y$ & $\Delta$ \\
0.0001 & -1.1087 & $0.4310^{-1}$ & 1.2413 & $0.8210^{-3}$ & 53.110 & $0.1410^{-4}$ \\
0.1 & 0.1646 & $0.1010^{-1}$ & 5.2635 & $0.1710^{-3}$ & 67.317 & $0.1210^{-4}$ \\
0.2 & 0.4071 & $0.2210^{-2}$ & 6.0654 & $0.2210^{-3}$ & 69.985 & $0.1110^{-4}$ \\
0.3 & 0.5963 & $0.2510^{-2}$ & 6.6627 & $0.2410^{-3}$ & 71.931 & $0.9510^{-5}$ \\
0.4 & 0.7708 & $0.5410^{-2}$ & 7.1866 & $0.2410^{-3}$ & 73.608 & $0.8310^{-5}$ \\
0.5 & 0.9465 & $0.7010^{-2}$ & 7.6884 & $0.2310^{-3}$ & 75.188 & $0.7210^{-5}$ \\
0.6 & 1.1361 & $0.7610^{-2}$ & 8.2023 & $0.2110^{-3}$ & 76.779 & $0.6010^{-5}$ \\
0.7 & 1.3565 & $0.7410^{-2}$ & 8.7664 & $0.1810^{-3}$ & 78.496 & $0.4910^{-5}$ \\
0.8 & 1.6397 & $0.6210^{-2}$ & 9.4462 & $0.1410^{-3}$ & 80.523 & $0.3610^{-5}$ \\
0.9 & 2.0826 & $0.4010^{-2}$ & 10.426 & $0.9010^{-4}$ & 83.367 & $0.2110^{-5}$ \\
0.9999 & 5.8365 & $0.7910^{-5}$ & 16.767 & $0.2810^{-6}$ & 99.863 & $0.5710^{-8}$ \\
\hline
\end{tabular}

\section{References}

1. M. Abramowitz and I.A. Stegun, Handbook of mathematical functions, Nat. Bur. Standards AMS 55, U.S. Govt. Printing Office, Washington, D.C. (1964).

2. O.E. Barndorff-Nielsen, Proc. Roy. Soc. London Ser. A, 353, 401-419 (1977).

3. A. Gil, J. Segura and N.M. Temme, Numerical methods for special functions, SIAM, Philadelphia, PA (2007).

4. G. Leobacher and F. Pillichshammer, Computing, 69, 291-303 (2002).

5. N.M. Temme, Math. Comp. 58, 755-764 (1992).

6. N.M. Temme, J. Comput. Appl. Math. 41(1-2), 145-157 (1992).

7. N.M. Temme, Special functions: An introduction to the classical functions of mathematical physics, John Wiley and Sons, New York (1996).

8. R. Wong, Asymptotic approximations of integrals, Classics in Applied Mathematics 34, SIAM, Philadelphia, PA (2001). 